\title{
Skin Aging Estimation by Facial Simulation
}

\author{
Yin Wu, Pierre Beylot, Nadia Magnenat Thalmann \\ MIRALab, CUI, University of Geneva \\ email: \{Yin.Wu, Pierre.Beylot, Nadia.Thalmann\}@cui.unige.ch
}

\begin{abstract}
We propose a layered facial simulation model for skin aging with wrinkles, which includes muscle, connective tissue and skin layer. Our aim is to simulate relevant facial animation and aging with the guidance of general facial tissue anatomy, so that the model can be extended to medical and cosmetic applications. B-spline muscle patches are automatically adapted to each individual face by mapping the anatomical facial muscle image. Connective tissues are simulated as simple springs with the length of hypodermis thickness that constrain skin movement. Facial skin deformation and aging are estimated based on an elaborated biomechanical model considering large strain deformation and wrinkle formation. Finally, multi-layered color and bump texture mapping are used to represent wrinkle forms and to render an aged face.
\end{abstract}

Keywords: Facial Simulation, Aging, Muscle Simulation, Wrinkle, Texture, Skin Deformation.

\section{Introduction}

Though being a small part of the human body, the face plays a significant role in human communication, emotion, identification and cosmetic. Facial simulation by computer is widely used in entertainment, teleconference and virtual reality.

Facial aging is a complex phenomenon with multiple factors. One of the main factors is skin aging with permanent wrinkles. Wrinkles hold significant characteristics on a human face; they are extremely important in the realism of facial simulation and aging. Two types of wrinkles appear with facial animation: expressive wrinkles and wrinkles due to age. Expressive wrinkles appear on the face during expressions at all ages and may become permanently visible over time. Facial skin changes with age: more lines and wrinkles emerge and the general appearance and texture of the facial skin become pronounced and rough. Permanent visible wrinkles are one of the major age indications of a person.

The reality of skin deformation and facial aging remains as an enigma in the fields of medicine and cosmetic. Facial simulation based on its soft tissue behaviors presents a strong potential in medical and cosmetic applications such as plastic surgery and aging studies. It is a challenge to realize part of the facial simulation and aging research by computer graphics.

\subsection{Facial simulation and aging with wrinkles}

Varied facial simulation models, such as geometric models and physically based models, have been developed for different purposes. There are many geometrical models, such as parametric model [Parke 74, 82], geometric operators [Waters 87] and abstract muscle actions [Magnenat-Thalmann 88]. There are also different kinds of physically-based models using either a particle system or a continuous system, such as the tension net model [Platt 81] and the three layered deformable lattice structure model ([Terzopoulos 90] and [Lee 95]). The finite element method is also employed for more accurate calculation of skin deformation, especially for potential medical applications ([Larrabee 86], [Pieper 92] and [Koch 96]).

There are a few efforts for facial wrinkle simulation. Viaud et al. have presented a geometric hybrid model for the formation of expressive wrinkles, where bulges are modeled as spline segments [Viaud 92]. There are also physically based facial animation models, where some wrinkles appear as the outcome of the skin deformation ([Terzopoulos 90] and [Wu 1994]). A dynamical wrinkle simulation system has also been developed, combining the physically based skin deformation with texture modeling of wrinkles ([Wu 1997]). It can be interesting to 
estimate aged faces with wrinkles on different individuals based on some convincing anatomy and biomechanics.

\subsection{Layered facial simulation model}

Our goal is to develop a facial simulation and aging model with wrinkles based on the facial anatomy and its soft tissues. A human face has a layered structure composed of a skull, then a muscle layer, which is covered by connective tissue and an outer skin layer. We have developed a physically-based facial animation and aging simulation model with a three-layered structure considering the soft facial tissues Figure 1. The deformation of skin is motivated by the simulated muscle layer, constrained by connective springs and decided by a biomechanical model. Our current research focuses on skin deformation, so that special attention is paid to elaborate the skin biomechanics and kinematics calculation. The muscle and connective tissue layers are simplified but powerful enough to provide relevant forces to skin.

Muscle contractions initiate all facial animations. Facial muscles are designed as B-spline patches according to the nature and direction of muscle fibers using the

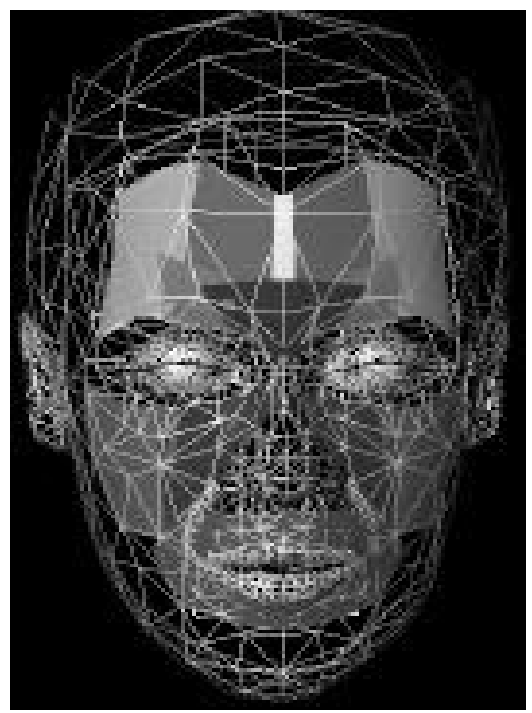

(a) Facial skin mesh and its muscle patches biaxial similarity between them. The muscles are constructed automatically on the facial skin surfaces with the same topology once a muscle set has been created on a generic face model. Connective tissues are simulated as a layer of springs with the length between the skin and the muscle layer.

In our facial model, the skin surface is represented as a triangle mesh, having material properties associated with triangles and kinematical features associated with vertices. Attention is being placed on the biomechanics of human skin in order to help explore aging reality and estimate individual aged face within different physical parameters. In addition to an elastic model considering large strain deformation, a plastic model is incorporated to calculate the permanent skin deformation, which provides deformation measures for wrinkle formation due to aging. In order to keep available force evaluation to surface triangulation of different size and shapes, the skin deformation force is determined on a triangular surface with a continuous system. The motion equations of skin are finally set up on vertices with a particle system.

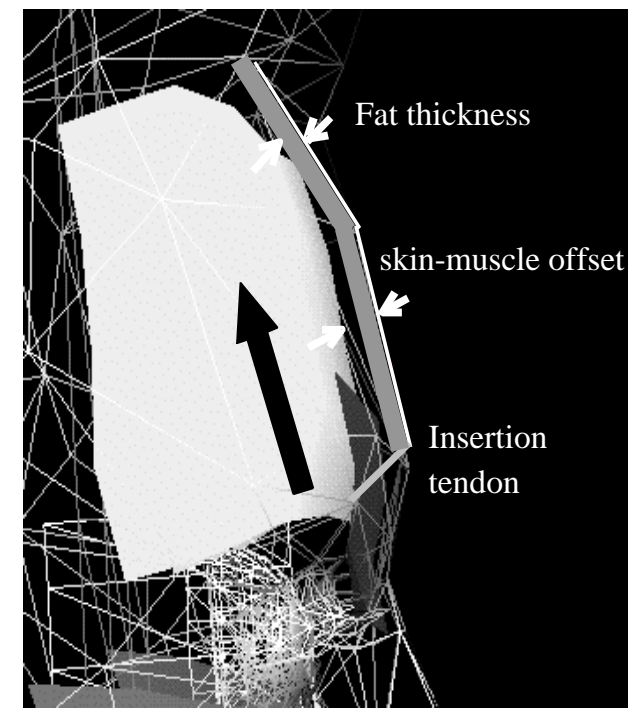

(b) Magnified side view

Figure 1: Layered facial simulation model

Beyond the facial layered structure to calculate facial animation, we use a texture model for skin rendering. The wrinkle formation and rendering details are represented by several layers of color, bump or displacement texture mapping. This facial model provides a strong potential in cosmetic and medical applications.

The rest of the paper describes our muscle simulation model, the skin deformation calculation and the wrinkle rendering approach. The concluding remarks are given at the end of the paper. 


\section{Muscle simulation}

Muscles are principle motivators of skin deformation so that when a muscle contracts, it attempts to draw its attachment together. As most facial muscles are flat and close to the skin surface, their shapes can be represented with the guidance of the skin surface. Our goal is not to model the exact muscle shapes but to provide relevant contracting forces to drive skin deformation for individual facial animation. B-spline patches are used to simulate muscles instead using complex volume models. However, the B-spline muscle patch is very much based on the anatomy facial muscle information and its construction is automated using a generic facial model.

\subsection{B-spline patch construction}

The biaxial feature of B-spline patches corresponds naturally to the facial muscle structure and behavior, so that the B-spline muscle patches activate the skin deformation with the advantage of simple geometric representations, director operations and relevant contracting force. The facial muscles are classified into two main types: linear muscles and sphincter muscles [Waters 87]. A linear muscle contracts toward the static attachment on the bone such as the frontalis major muscle that raises the eyebrows. A sphincter muscle contracts around an imaginary central point such as the orbicularis oris muscle which draws the mouth together. Open B-spline patches are used to simulate the linear muscles while the B-spline patches closed in one parametric dimension are employed to imitate the sphincter muscles (Figure 2). B-spline muscle patches are constructed according to the muscle fiber nature. While one parameter direction of a muscle patch approximates the muscle fiber orientation, intuitively, the transverse parameter dimension indicates the potential wrinkle locations during skin deformation. Muscle contractions are simply simulated by scaling the B-spline patch along $v$ parameter direction.

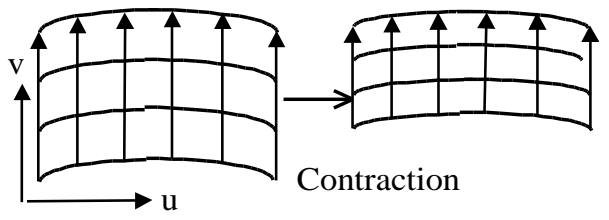

(a) Linear muscle: Open Bspline patch

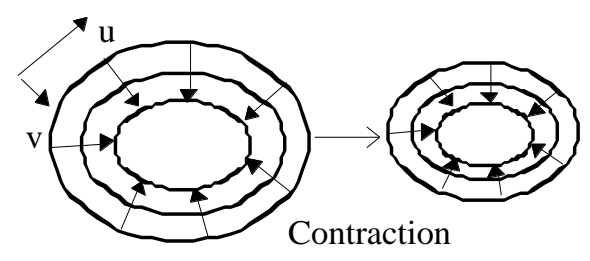

(b) Sphincter muscle: Close B-spline patch

Figure 2: Muscle Operations

\subsection{Automatic muscle construction using anatomic muscle mapping}

In our facial system, a muscle patch not only drives the skin deformation but also provides the directions of principal skin deformation, which guide the skin deformation calculation. Though the muscle layer is an inner layer in our facial simulation model, it is very important to construct the B-spline muscle patches corresponding to the real facial anatomy in order to achieve the realism of facial simulation. We use a simple but efficient anatomic muscle mapping approach to solve this problem.

Figure 3 (a) shows an anatomic muscle image with a mesh of lines to mark the ideal B-spline muscle patches that should be designed on it. Using a texture mapping tool, a photograph can be mapped on a face by imposing position correspondence between the features of the model and the image ([Kalra 93] and [Sannier 97]). Feature points such as in Figure 3 (b) can be used to map the photographs as well as an anatomy facial muscle image on the 3D face model. The TextureFit tool developed in MIRAlab ([Sannier 97]) is used to define the feature points interactively on one 3D face model and the anatomic facial muscle image. The whole anatomic facial muscle image is then mapped automatically to the 3D face as a texture image Figure 3 (c). The anatomic image can be automatically mapped to another 3D face model with the same surface topology without the need to specify feature points interactively. 


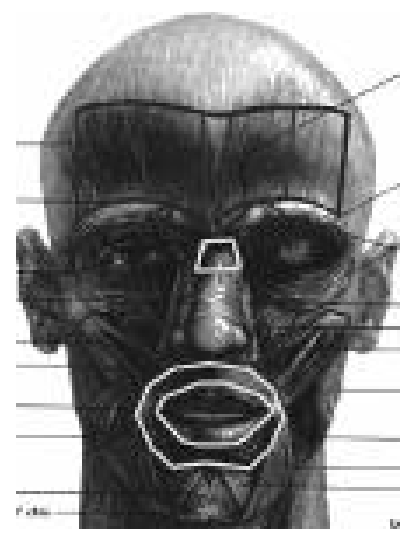

(a) An anatomy muscle image mapped

marked with patch mesh

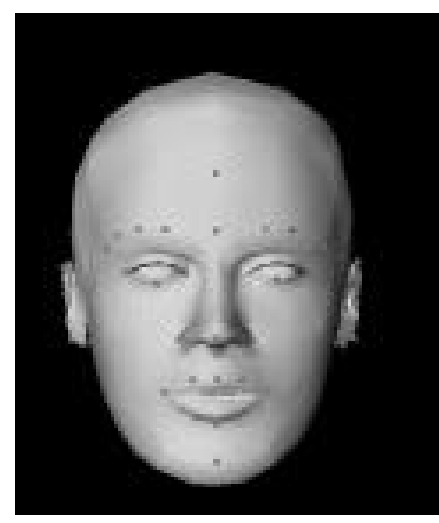

(b) 3D face model with feature

points for texture mapping

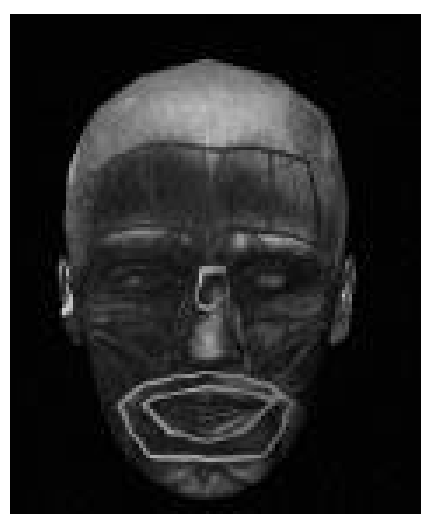

(c) 3D face model

\section{by facial muscles}

Figure 3: Automatic muscle adaptation by anatomy facial muscle mapping

The positions of the key points to construct the facial muscles are marked once on the anatomy muscle image (Figure 3(a)). Suppose (u, v) is the image position at one of the key point in the anatomy image. Given an individual face model, all the texture coordinates $(\mathrm{s}, \mathrm{t})$ of the facial surface vertices are automatically calculated using the texture mapping tool. A triangular mesh is constructed in the $2 \mathrm{D}$ texture space. The key point $(\mathrm{u}, \mathrm{v})$ can be located inside one of the triangles in the 2D texture space. Its barycentric coordinate values inside the triangle are calculated. Each triangle in the 2D texture space has a corresponding triangle on the $3 \mathrm{D}$ face. The 3D position of this key point can be obtained by linear interpolating the three vertex positions using its barycentric coordinates in the texture triangle. The facial muscle patches are automatically constructed by B-spline interpolation once the 3D key point positions of each muscle are calculated. Figure 4 shows a set of facial muscles automatic generated on a face using this approach.
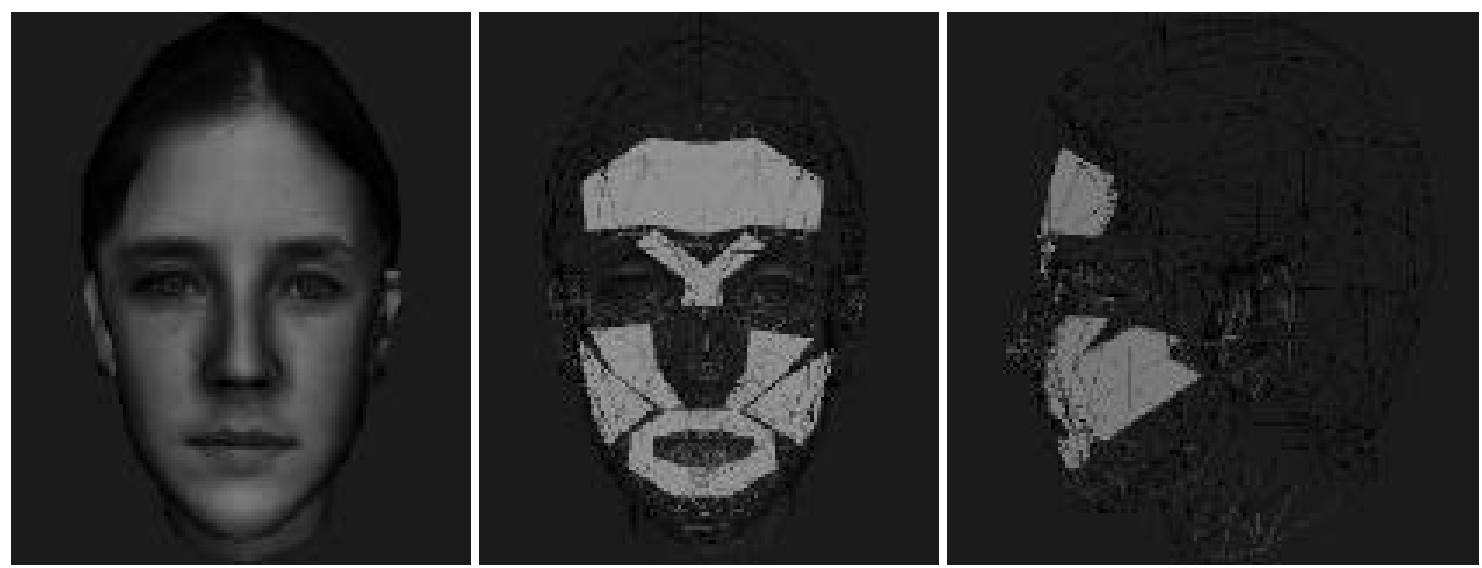

Figure 4: Automatic generated muscle sets on a face

\section{Skin deformation and aging model}

Different biomechanical models of skin have been proposed in the literature [Lanir 87]. As a biological material, skin is described in a material continuum employing the concepts that are different to particle physics. The stress-strain relationship is a common and direct way to describe the elasticity of skin. The main idea to calculate skin deformation model is to determine the forces on a triangular surface with a continuous system while the motion equations are set up on vertices 
with a particle system. Therefore, the force evaluation is available to the surface triangulation of different size and shapes, while the motion equations of the particle system remain simple and efficient, which has a direct impact on the kinematics parameters of vertices.

The deformation model is developed based on a biomechanical skin model undergoing large deformation [Danielson 75]. The skin is considered to be biaxial and incompressible and of constant thickness at each region. It does not support negative stress, and hence buckles easily. As skin is quite thin and flexible, a plane stress elastic model is applied for each triangle.

There are three steps to reach the skin deformation calculation. Firstly, we analyze the skin deformation to calculate the strain stress and the internal force. All types of forces are then accumulated and the deformation equations are to be relevantly set and solved. Finally, a plastic model is used to calculate the wrinkle formation on skin

\subsection{Internal force analysis on skin surface}

To analyze the internal force on skin deformation, the internal strain of a deformed triangle on skin membrane is first measured. A two-phase linear stress-strain relationship is used for computing the stress components on the triangle and internal forces are finally accumulated on vertices.

\subsubsection{Small strain deformation on skin surface}

In the case of small strain deformation, any three measurements of strain in a plate called "strain rosette" [Timoshenko 82], is enough for completely evaluating the strain. With a convenient local coordinate system $(x, y)$, a set of three strain measurements can be used to calculate the components of strain vector $\left(\varepsilon_{x}, \varepsilon_{y}, \gamma_{x y}\right)$.

Usually, a local coordinate system is set up with the $X$ axis along one triangle edge (Figure 5), and the other two edges have angles of $\theta i$ with the $X$ axis. Each edge of the triangle is taken as a strain gauge giving strain measurement on the skin surface.
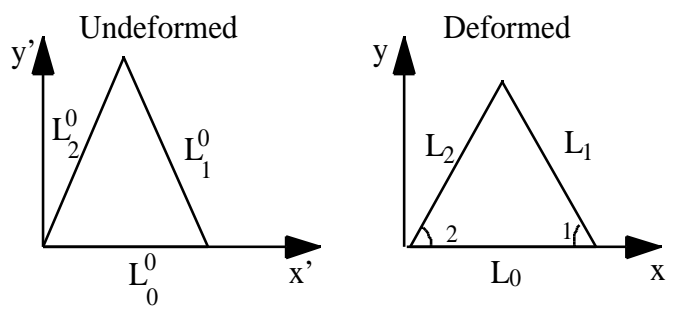

Figure 5: Triangle deformation in a local coordinate system

\subsubsection{Large strain deformation on skin surface}

In the case of large strain deformation, this "strain rosette" method is no longer valid because the formula is derived under the condition of small deformation [Timoshenko 82]. It can result strain vectors having component out of the strain bound $(-1, \infty)$. The general theory of large deformation is rather complicated to apply because of the non-linearity of between the strain and displacement.

Nevertheless, the incompressibility of skin simplifies the problem. The principal strain and stress directions can be estimated since the deformation moves along a uniform direction at each point. The principal strain direction is along a line on the membrane with no shear strain component at a deformed state.

In Danielson's biomechanical skin model for the Zplastic operation [Danielson 75], the large deformation is analyzed by studying the stress and strain along the tension ray, in other words, along the direction of maximum stress. As there is no shear deformation along these directions, the strain can be easily measured. Then the stress-strain relationship can be used to directly calculate the stress. This theory is applied to the plane sheet having an elliptical hole, which is closed into a straight line coinciding with its major axis ( Figure 6).

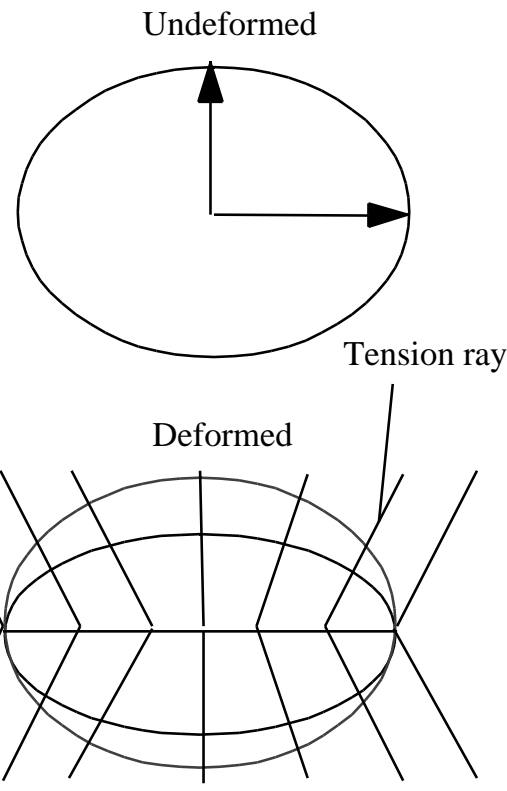

Figure 6 : Skin deformation within an elliptical hole 
We apply this theory with triangle surfaces undergoing large deformation. The simplification of the strain vector evaluations is based on the incompressibility of skin and the uniformity of deformation directions controlled by muscle contraction at each skin region.

To calculate the strain vector in large deformation, the principal strain directions of a triangle are first estimated. Guided by muscle contraction, the skin deformation follows the direction of the muscle contraction. Therefore, the shear strain on skin surface is very small along the direction of muscle contraction, the direction of $v$ parameter on the B-spline muscle patch. It is calculated as the B-spline surface derivative with respect to $v$ at each muscle point. The principal strain direction with maximum deformation at a skin vertex is approximated by the contraction direction at its connective muscle point, while the direction orthogonal to the strain direction approaches the potential wrinkle line. Figure 7(a) shows the estimated principal strain direction of maximum deformation on one side of a face, while the muscle meshes are displayed on the other side of the face as a reference. The principal direction of a triangle is calculated as an average value of the directions of its vertices.

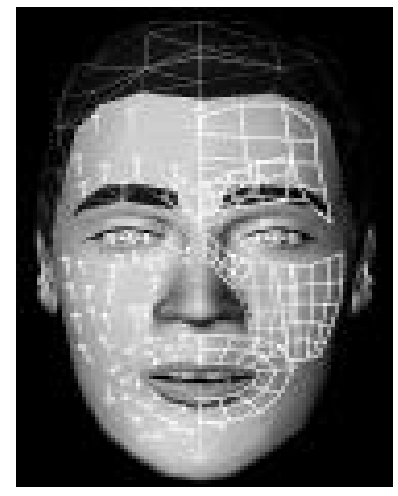

(a) Principal deformation direction

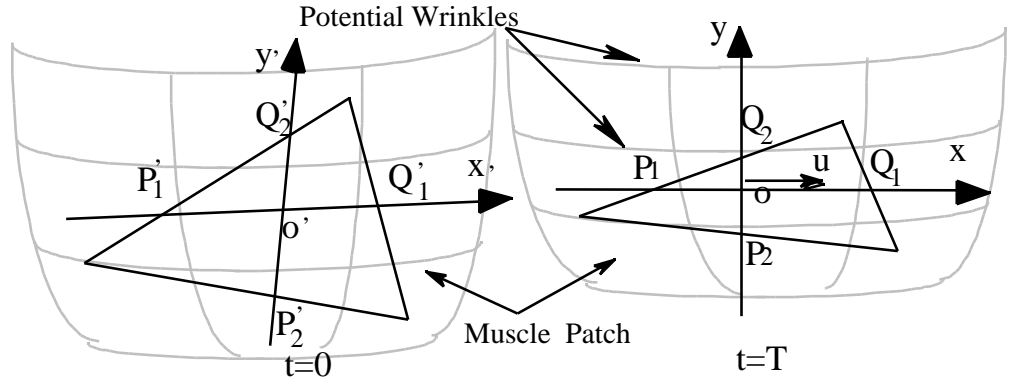

(b) Local coordinate system for a triangle

Figure 7 : Skin principal deformation directions \& local coordinate system for a triangle

The strain vector is then calculated in the local coordinate system, whose axes approach to the principal strain directions. Each triangle employs a local coordinate system $(X, Y)$ according to the principal direction. Generally, the $Y$ axis corresponds to the direction of muscle contraction, and the $X$ axis to the potential wrinkle lines (see Figure 7 (b)). The potential wrinkle line is assumed remaining vertical to the muscle contraction direction in the deformed state.

The local coordinate system is set up in the deformed triangle, as the forces are calculated with respect to the current state at each step $T$. To represent the potential wrinkle direction during deformation, a wrinkle segment $P_{1}^{\prime} Q_{1}^{\prime}$ is defined for each triangle in the initial state. Having the estimated principal deformation direction of each triangle shown in Figure 7 (b), the wrinkle segment is set as a line vertical to this direction through centroid O' of the triangle at initial state. The locations of $P_{1}^{\prime}$ and
$Q_{1}^{\prime}$ are represented as the ratios by which they cut respectively two of the three triangle edges, so that they can be referenced in a deformed state. Under this assumption, the strain vector is directly calculated by measuring strain along the local $X$ coordinate axis.

The large strain evaluation is applied to triangles whose principal deformation directions can be estimated from muscle contraction direction. For these triangles on skin without connection to muscles the principal directions can not be estimated. However these triangles are in the region with smaller deformation, and it is still proper to consider them using the small strain calculation.

\subsubsection{Stress and internal force}

A two-phase linear stress-strain relationship is employed to describe skin's elasticity. After the strain components have been calculated, Hook's law is used for computing the components of stress vector 
$\left(\sigma_{x}, \sigma_{y}, \tau_{x y}\right)$ on a triangle. The principal stress components are calculated along the two vertical directions according to the formula of calculating the stress of any point along certain direction [Timoshenko 82]. The negative component is either neglected, or reduced into an insignificant value while the other components are used for calculating the in-plane forces along triangle edges.

The stress and strain vectors of triangles describe the dynamics of skin deformation in a continuous space. This provides the facility to evaluate the internal force considering the spatial extent of a triangle element. However, since the motion equations are set up on vertices, the in-plane stress of triangles needs to be converted to the vertices as a force component. The stress components on a triangle are converted into inplane forces along its edges. Finally each edge force is distributed to its two vertices [Volino 95].

\subsection{Deformation solution}

The aim is eventually to determine the deformed surface shape responding to the forces calculated from the mechanical skin model. In our approach, Newtonian mechanics, governed by the law $F=m a$, is used to describe the dynamics of surface deformation on vertices. Forces contributing to motion are divided into three categories: internal elastic force, external spring forces and viscosity force.

Intuitively, the sum of forces is represented as: $F=F_{\text {int }}+F_{\text {ext }}+F_{\text {visco }}$.

where:

$F_{\text {int }}$ - the internal force distributed to the vertex

$F_{\text {ext }}$-- the external force distributed to the vertex

$A-$ area around the vertex influenced by the force

$\rho-$ mass density of skin

However, it doesn't produce correct deformation using this method fed with the real skin parameters. A study on internal and external forces manifests the different influence region of the two types of forces. The internal force $F_{\text {int }}$ derived from elastic stress corresponds to the triangle boundaries, as the internal forces inside the triangle are eliminated by stresses on the two opposite sides of the skin cross section. Therefore, the internal force $F_{\text {int }}$ on a vertex only influence the motion of the triangle boundaries. The mass of the triangle edges connecting the vertex is summarized as the mass factor in Newton's second law. A more accurate evaluation of acceleration is:

$$
a_{\mathrm{int}}(t)=\frac{F_{\mathrm{int}}}{\rho \cdot L_{j}}=\frac{\sum_{j} L_{j} T_{s^{*}} f\left(\sigma_{x}, \sigma_{y}, \tau_{x y}\right)}{\rho \cdot \sum_{j} L_{j}}
$$

where: $\sum_{j} L_{j}--$ the sum of the edges connecting to the vertex.

On the contrary, the external spring forces are applied to the whole neighboring region around the vertex. The acceleration can be expressed as:

$$
a_{\text {ext }}(t)=\frac{F_{\text {ext }} \cdot \text { Area }}{\rho \cdot \text { Area } .}=\frac{F_{\text {ext }}}{\rho}
$$

The acceleration of viscoelastic component is represented as:

$$
a_{\text {visco }}(t)=-\gamma \frac{v(t)}{\rho}
$$

where: $\quad \gamma-$ the damping constant.

The total acceleration value is the sum of the three components:

$$
a(t)=a_{\mathrm{int}}(t)+a_{\text {extt }}(t)+a_{\text {visco }}(t)
$$

The resultant of acceleration is calculated by adding up these two acceleration components. For an evenly triangularized mesh, the independence of mesh size is verified with the equilateral triangle example. Although this evaluation of acceleration is not as accurate as Finite Element Analysis, it does give a reasonable motion evaluation for the deformation surface. The importance of this correction is proved especially when real mechanical parameters can be applied to the model instead of using artificial ones.

\subsection{Expressive wrinkle and aged wrinkles measurement}

\subsubsection{Expressive wrinkles}

Due to the incompressibility of the skin, skin buckles and bulges appear under negative strains. A simple mapping approach is used for obtaining the appropriate height value of the bulge function from the deformation or strain value. Suppose the reference triangle of a point on the wrinkle line has a deformation of compression strain $\varepsilon$. The original length is $2 l_{0}$; after deformation it is 21. A bulge is formed on the line because of the incompressibility. The deformation, the bulge height $d$ is 
approximated as (assuming linear case for bulge) $\mathrm{I}_{0}^{2}=\mathrm{I}^{2}+\mathrm{d}^{2}$. The height of the bulge function is computed as a function of strain and bandsize. During the animation, the height function changes accordingly to the strain value.

\subsubsection{Aged wrinkles}

Plasticity causes the irreversible atom dislocation and permanent deformation beyond certain force limits. The remaining deflection of the skin after the removal of load is the result of plasticity. Shanders [Shanders 73] proposed a plasto-visco-elastic skin model in which the total deformation is the sum of an initial elastic response, a viscous component and a plastic component. Under a constant deformation, the plastic component linearly depends on the load duration. This model is extended to present the sum of plastic deformation after a whole deformation process:

$$
V_{p}=\int_{0}^{T} V^{T} e^{(t) d t}
$$

where:

$V_{p}-$ the permanent deformation after animation

$V e(t)$ - the deformation at time $t$ during the animation

$T-$ the animation duration

In our approach, the plastic deformation is applied to wrinkle bulge height. Age wrinkles are visible after repeated skin deformation over years. The deformation during facial expression is recorded by the wrinkle bulge height $d$. The bulge height of a permanent wrinkle, $d_{p}$, is calculated as the deformation $V_{p}$ in the plastic model described as:

$$
d_{p}=\underset{i}{a} d_{i} \Delta t_{i}
$$

where: $\quad d_{i}-$ the bulge height at step $i$

$\Delta t_{i}-$ the time duration at step $i$

$a$-- the parameter of the degree of

permanent deformation.

Wrinkles are finally generated and rendered dynamically by modifying texture image, which will be described in the following part.

\section{Wrinkle rendering and results}

As an outer most visible layer, the modeling and rendering of skin is very important in the realism of the facial animation. It is quite expensive to geometrically model all the wrinkles on the 3D skin surface. This would require very fine skin surface resolution resulting in enormous computation. Thus the dynamics of wrinkle simulation is computed using the strain measures from skin deformation of the 3D facial model, where the facial skin mesh is deformed according to the biomechanical skin model described previously. The information from skin deformation modifies the corresponding texture image of synthetic patterns and generates wrinkles.

Wrinkles are produced corresponding to the facial skin movement controlled by muscle contractions. While one parameter direction of a muscle patch approximates the muscle fiber orientation, intuitively, the transverse parameter dimension indicates the potential wrinkle locations with skin deformation. When the facial muscles are constructed, the wrinkle location can be defined. Wrinkle can also be automatically located with the help of some feature points on face [Lee 99]. Texture image of wrinkles are modified during skin deformation, multilayered color and bump texture mapping are used for photo realistic rendering. Some face shape change can also be considered, either by shape change on some facial regions such as on cheeks, or by interpolating the shape slightly to an old model. Figure 8 shows some of the results. 

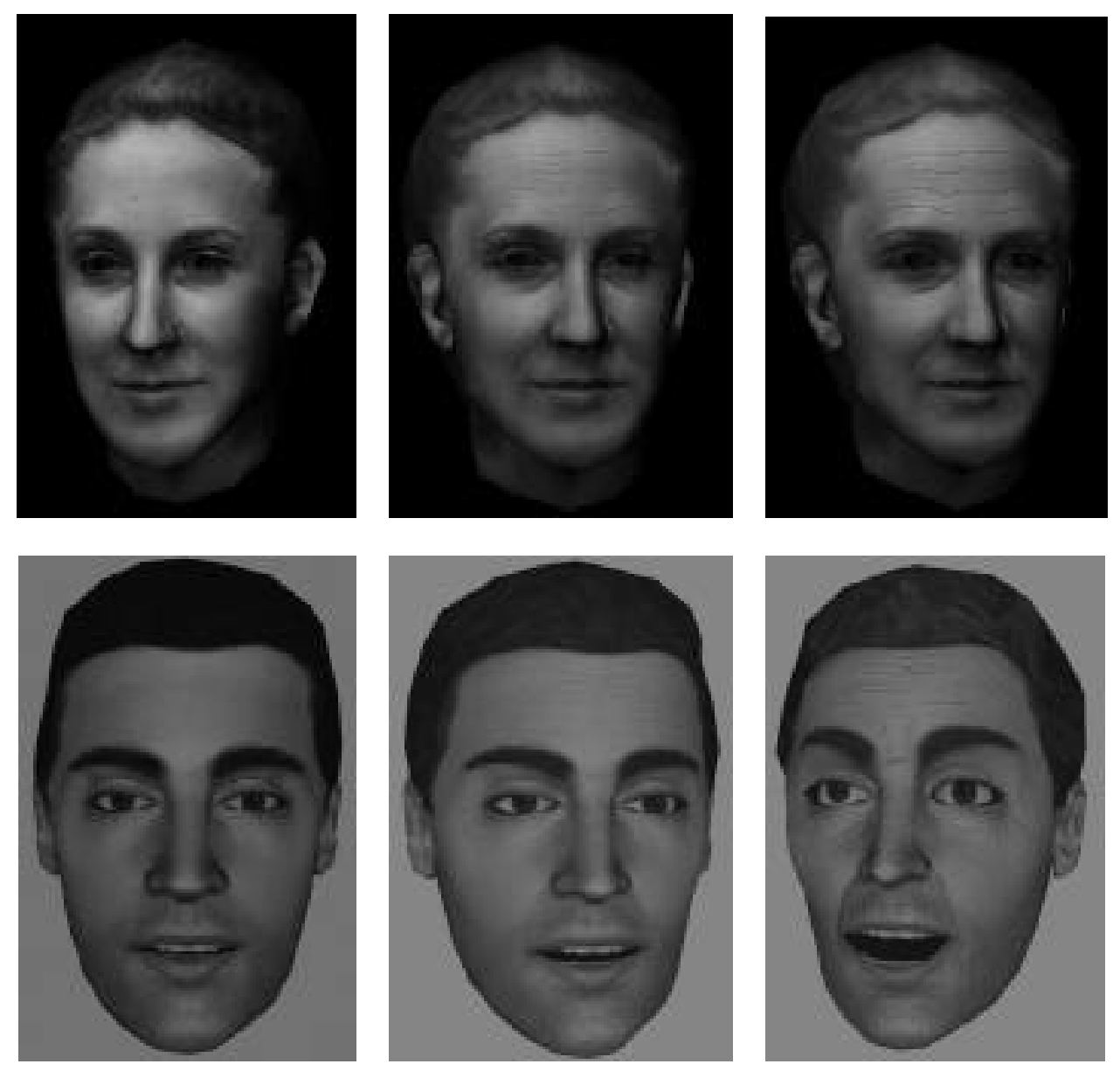

Figure 8 : Aged faces

\section{Conclusion}

In this paper we have introduced a facial simulation aging model with wrinkles. Facial muscles are constructed automatically on an individual face based on anatomic image mapping. Skin deformation employs biomechanical model and the solution of deformation is elaborated to obtain the accuracy. Wrinkles and other details of the skin are designed on texture images. The skin deformation strain is used to form expressive wrinkles and finally to result age wrinkles using plasticity. The aged face with wrinkles can be estimated for individuals. Our biomechanical model is suitable for applications that require acceptable accuracy and an interactive display. It can be a potential tool for aging studies, facial surgery planning.

\section{Acknowledgment}

This research is supported by the Swiss National Research Foundation(FNRS). The authors would like to thank Gael Sannier to provide the face texture mapping tool. Thank-you also to Wonsook Lee for a lot of discussion in wrinkle generation.

\section{References}

Danielson D A (1973), "Human Skin as an Elastic Membrane, " J.Biomechanics. Vol.6,pp.539-546, Elsevier Science.

Danielson D A and Natarajan S (1975), "Tension Field Theory and the Stress in Stretched Skin," J. Biomechanics, Vol .8, pp. 135-142, Elsevier Science.

Elden H R (1977), Biophysical Properties of Skin, Ed. WileyInterscience, New York.

Farin, G (1990), Curves and Surfaces for Computer Aided Geometric Design, A Practical Guide, Academic Press, Second Edition. 
Ishii T, Yasuda T, Yokoi S and Toriwaki J (1993), "A Generation Model for Human Skin Texture," Proc. of CGI '93, pp. 139-150, IEEE Proceedings.

Gritz L, BMRT Home Page: http://ww.seas.gwu.edu/students/gritz/bmrt.html.

Kalra P, Mangili A, Magnenat-Thalmann N, Thalmann D (1992), "Simulation of Facial Muscle Actions Based on Rational Free Form Deformations," Proc. Eurographics‘92, pp. 59-69, NCC Blackwell.

Kalra P and Magnenat-Thalmann N (1993), "Simulation of Facial Skin using Texture Mapping and Coloration," Proc. ICCG'93, Bombay India, in Graphics, Design and Visualization, pp. 247-256.

Kaufman A (1988), "TSL- a Texture Synthesis Language," The Visual Computer, Vol. 4, No. 3, pp. 148-158, Springer Verlag.

Koch RM, Gross MH, Carls FR, Von Buren DF, Fankhauser G and Parish YI(1996), "Simulation Facial Surgery Using Finite Element Models," Proc. of SIGGRAPH'96, Computer Graphics, pp. 421-428.

Lanir, Y (1987), "Skin Mechanics," in Ed. Skalak, R. Handbook of Bioengineering, Mc GrawHill Book Company.

Larrabee WF(1986), "A Finite Element Method of Skin Deformation: I. Biomechanics of Skin and Soft Tissues," Laryngoscop, 96: 399-405.

Lee W, Wu Y and Magnenat-Thalmann N(1999) "Cloning and Aging in a VR Family”, accepted to IEEE VR'99, 1999.

Lee Y and Terzopoulos D(1995), "Realistic Modeling for Animation," Proc. of SIGGRAPH'95, Computer Graphics, pp. 55-62.

Magnenat-Thalmann N, Primeau E, Thalmann D (1988), "Abstract Muscle Action Procedures for Human Face Animation," The Visual Computer, Vol. 3, No. 5, pp. 290-297, Springer Verlag.

Miller, G (1988), "The Motion Dynamics of Snakes and Worms," Proc. of SIGGRAPH'88, Computer Graphics, Vol. 22, No. 4, pp. 169-178.

Nahas M, Huitric H, Rioux M and Domey J (1990), "Facial Image Synthesis Using Skin Texture Recording," The Visual Computer, Vol. 6, pp. 337-343, Springer Verlag.

Parke FI(1974), "A Parametric Model for Human Faces," Ph. D Dissertation, University of Utah.

Parke FI(1982), "Parametric Model for Facial Animation," IEEE Computer Graphics and Applications, Vol. 2, No. 9, pp. 61-68.

Platt S, Badler N(1981), "Animating Facial Expressions," Proc SIGGRAPH'81 Computer Graphics, Vol. 15, No. 3, pp. 245252.

Sannier G, Magnenat-Thalmann N(1997), "A User-Friendly Texture-Fitting Methodology for Virtual Humans," Computer Graphics International'97, pp 167-176, Springer Verlag.
Pieper S(1992), " CAPS: Computed-Aided Plastic Surgery," Ph.D Thesis, MIT, Media Arts and Sciences, Cambridge, MA.

Shanders R(1973), "Torsional Elasticity of Human Skin in Vivo," Pluegers Arch., No.342, pp. 255-260.

Terzopoulos D, Waters K(1990), "Physically-Based Facial Modeling and Animation", Journal of Visualization and Computer Animation, Vol. 1, pp. 73-80, John Wiley \& Sons.

Upstill S(1989), The RenderMan Companion, AddisonWesley.

Viaud M, Yahia H (1992), "Facial Animation with Wrinkles," 3rd Workshop on Animation, Eurographics'92, Cambridge, Springer Verlag.

Waters K(1987), "A Muscle Model for Animating Three Dimensional Facial Expression," Proc SIGGRAPH'87, Computer Graphics, Vol.21, No. 4, pp. 123-128.

Wu Y, Magnenat Thalmann N and Thalmann D (1994), "A Plastic-Visco-Elastic Model for Wrinkles In Facial Animation And Skin Aging," Proc. Pacific Conference '94, pp.201-213, World Scientific.

Wu Y, Kalra P and Magnenat Thalmann (1997), "Physicallybased Wrinkle Simulation \& Skin Rendering," Eurographics Workshop on Animation and Simulation, pp. 69-79, Springer Verlag. 